\title{
Direct Imaging of Hydrogen Atoms in a Crystal by Annular Bright-Field STEM
}

\author{
E. Abe, ${ }^{*}$ R. Ishikawa, ${ }^{*}$ E. Okunishi, ${ }^{* *}$ H. Sawada, ${ }^{* *}$ Y. Kondo, ${ }^{* *}$ and F. Hosokawa** \\ *Department of Materials Science \& Engineering, University of Tokyo, Tokyo 113-8656, Japan \\ **Electron Optics Division, JEOL Ltd., Tokyo 196-8558, Japan
}

Enhancing the imaging power of microscopy to identify all chemical types of atom, from low- to high-atomic-number elements, would significantly contribute for a direct determination of material structures. Scanning transmission electron microscopes (STEM) successfully provided images of heavy atom positions by annular dark-field method, but detection of light atoms was difficult owing to their weak scattering power. For detection of lighter atoms with extremely weak scattering, annular-bright-field (ABF) phase-contrast imaging may be preferred, since it requires the atoms only to alter a phase of wave. In fact, a significant sensitivity of ABF-STEM was demonstrated by imaging oxygen ${ }^{1,2}$ and nitrogen ${ }^{1}$ atoms in crystalline solids, and further lithium atoms in a $\mathrm{LiV}_{2} \mathrm{O}_{4}$ compound $^{3}$. In the present work, using ABF-STEM we demonstrate the direct imaging of the lightest hydrogen atoms in a crystalline solid ${ }^{4}$.

The optimum conditions of ABF-STEM imaging can be derived based on phase-contrast transfer function (PCTF) of the hollow-cone illumination (HCI) TEM. Reciprocity supports the equivalence between the techniques (Fig. 1) and practically holds within a weak-phase approximation. The HCI is able to improve significantly the resolution as well as signal-to-noise ratio of a phase-contrast by eliminating the effect of wavelength fluctuations of the incident beam, i.e., a chromatic aberration $\left(\mathrm{C}_{\mathrm{c}}\right)$ that causes the focus instability. It was also shown that, compared with the axial illumination, the achievable resolution of $\mathrm{HCI}$ can be almost doubled with the optimized conditions derived by PCTF analysis. The fundamentals of HCI-related TEM/STEM imaging were established as the Rose-Cowley method by their pioneering works. Practically, ABF-STEM may be advantageous rather than dynamic HCI-TEM, which requires continuous and precise beam-locking illuminations.

Optimized HCI condition ( $11 \mathrm{mrad} \leq \theta_{\mathrm{c}} \leq 22 \mathrm{mrad}, \theta_{\mathrm{c}}$ : cone-angle) derived from the $\mathrm{C}_{\mathrm{s}}$-corrected microscope parameters (JEM-ARM200F, operated at $200 \mathrm{kV}$ ) confirms that the first-zero of PCTF can be extended into $22.5 \mathrm{~nm}^{-1}$, which corresponds to a spatial resolution of about $44.4 \mathrm{pm}^{4}$. This is readily realized as the ABF-STEM condition with $11 \mathrm{mrad} \leq \theta_{\mathrm{d}} \leq 22 \mathrm{mrad}\left(\theta_{\mathrm{d}}\right.$ : detector-angle), leading to a successful imaging of the hydrogen atom columns in a crystalline compound $\mathrm{YH}_{2}$ (Fig. 2). Similar observation has been also demonstrated for the $\mathrm{VH}_{2}$ hydride 5 .

References

[1] E. Okunishi et al., Microsc. Microanal. 15 (2009) 164.

[2] S.D. Findlay et al., Appl. Phys. Lett. 95 (2009) 191913.

[3] Y. Oshima et al., J. Electron Microsc. 59 (2010) 457.

[4] R. Ishikawa et al., Nature Materials doi:10.1038/nmat2957 (2011).

[5] S.D. Findlay et al., Appl. Phys. Express 3 (2010) 116603.

[6] This work is partly supported by a Grant-in-Aid for Scientific Research on Priority Areas "Atomic Scale Modification" from the Ministry of Education, Culture, Sports, Science and Technology of Japan (MEXT). 


\section{HCI-TEM}

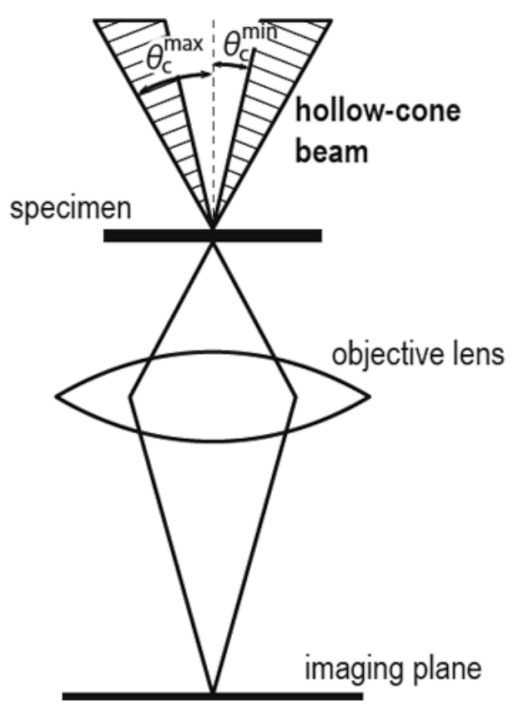

\section{ABF-STEM}

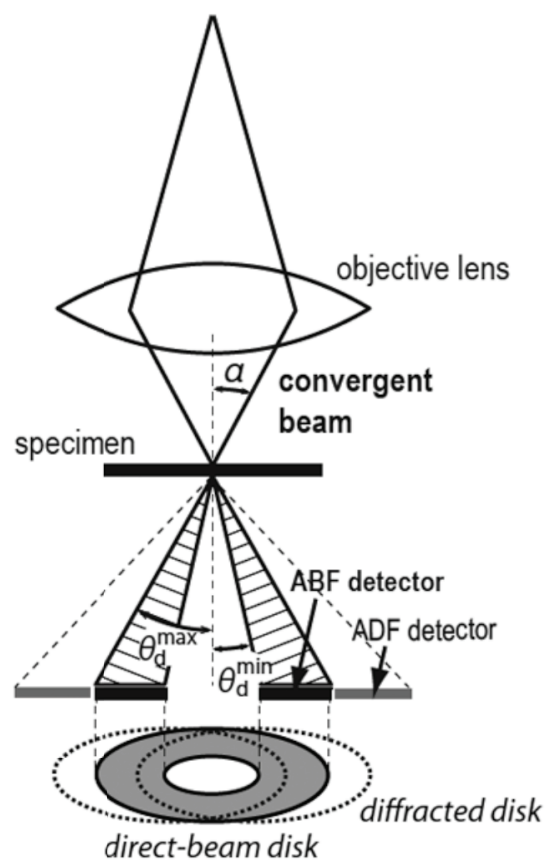

FIG. 1. Schematic ray diagrams of HCI-TEM and ABF-STEM according to reciprocity.
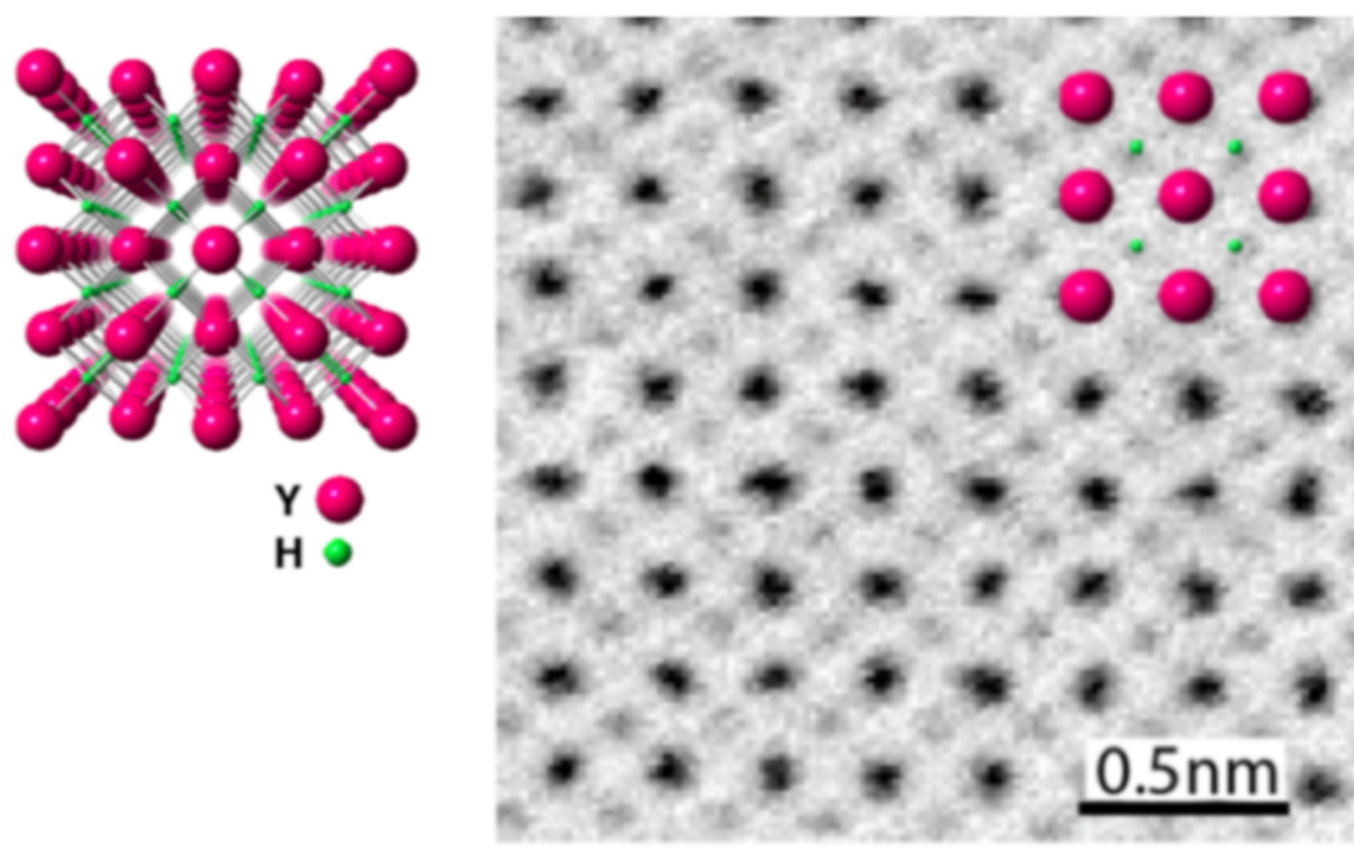

FIG. 2. ABF -STEM image of the crystalline compound $\mathrm{YH}_{2}$, viewed from the [010] direction. $\mathrm{YH}_{2}$ unit-cell projection is inserted in the image. 